\title{
Novos e raros registros de Euglenophyta incolores na Planície Costeira do Rio Grande do Sul, Brasil
}

\author{
Sandra Maria Alves-da-Silva ${ }^{1,2}$ e Francieli Friedrich ${ }^{1}$
}

Recebido em 05/11/2007. Aceito em 31/03/2009

\begin{abstract}
RESUMO - (Novos e raros registros de Euglenophyta incolores na Planície Costeira do Rio Grande do Sul, Brasil). São apresentados nove táxons de Euglenophyta incolores como resultado do estudo taxonômico em ambientes lênticos (lagoas, açude, canal e banhados) de duas áreas próximas a Laguna dos Patos: a Lagoa do Casamento e ecossistemas associados $\left(30^{\circ} 03^{\prime}-30^{\circ} 34^{\prime} \mathrm{S}\right.$ e $\left.50^{\circ} 25^{\prime}-50^{\circ} 47^{\prime} \mathrm{W}\right)$ e região do Butiazal de Tapes (30 $23^{\prime}-$ $30^{\circ} 38^{\prime} \mathrm{S}$ e $51^{\circ} 16^{\prime}-51^{\circ} 29^{\prime} \mathrm{W}$ ) na Planície Costeira do Rio Grande do Sul. As coletas de rede e espremido de macrófitas aquáticas abrangeram as estações de outono e primavera de 2003. Quanto à distribuição todos os nove táxons de Euglenophyta incolores são novas citações para a Planície Costeira do Estado, sendo primeiros registros para o Brasil: Cyclidiopsis acus Korsch., Entosiphon polyaulax Skuja, Gyropaigne kosmos Skuja, Menoidium tortuosum (Stokes) Lemm. var. playfairii Bour. e Rhabdomonas mirabilis (Playf.) Schroeckh, Lee \& Patterson, e ainda para o Rio Grande do Sul: Menoidium gracile Playf., Menoidium pellucidum Perty e Menoidium obtusum Pringheim. O ecossistema banhado foi o que apresentou maior riqueza destacando-se o banhado entre a Lagoa do Capivari e Lagoa do Casamento por ter sido encontrado sete táxons dentre os nove identificados. A área da Lagoa do Casamento apresentou maior riqueza de Euglenophyta incolores do que os ambientes próximos ao Butiazal de Tapes, possivelmente por esta área possuir maior contribuição antrópica.
\end{abstract}

Palavras-chave: Euglenophyta despigmentadas, taxonomia, sul do Brasil

ABSTRACT - (New and rare reports of colorless Euglenophyta on the Coastal Plains, Rio Grande do Sul state, Brazil). Nine taxa of colorless Euglenophyta are presented as a result of a taxonomic study in lentic environments (lagoons, wetlands, pond, and canal) of two areas near Patos Lagoon: Casamento Lake and associated ecosystems $\left(30^{\circ} 03^{\prime}-30^{\circ} 34^{\prime} \mathrm{S}\right.$; $\left.50^{\circ} 25^{\prime}-50^{\circ} 47^{\prime} \mathrm{W}\right)$ and the Butiazal de Tapes region $\left(30^{\circ} 23^{\prime}-30^{\circ} 38^{\prime} \mathrm{S}\right.$; $\left.51^{\circ} 16^{\prime}-51^{\circ} 29^{\prime} \mathrm{W}\right)$ on the Coastal Plains of Rio Grande do Sul. Sampling with nets and pressed macrophytes were carried out during fall and spring 2003. All nine taxa are new reports for the Coastal Plains of the state, where Cyclidiopsis acus Korsch. Entosiphon polyaulax Skuja, Gyropaigne kosmos Skuja, Menoidium tortuosum (Stokes) Lemm. var. playfairii Bour. and Rhabdomonas mirabilis (Playf.) Schroeckh, Lee \& Patterson are first reports for Brazil, and Menoidium gracile Playf., Menoidium pellucidum Perty and Menoidium obtusum Pringheim for Rio Grande do Sul. The wetland ecosystem had the highest richness, where seven of the nine identified taxa were found in the wetlands between Capivari and Casamento lakes. The Casamento Lake area had higher richness of colorless Euglenophyta than the habitats in the Butiazal de Tapes region, probably because Casamento Lake received a higher anthropic contribution.

Key words: Colorless Euglenophyta, taxonomy, southern Brazil

\section{Introdução}

O estudo das Euglenophyta fez parte de um programa mais amplo, o "Projeto de Conservação e Utilização Sustentável da Diversidade Biológica Brasileira - PROBIO", cujo objetivo foi realizar o levantamento da flora e fauna em duas áreas localizadas próximas à Laguna dos Patos: a Lagoa do Casamento e a região do Butiazal de Tapes na Planície Costeira do Rio Grande do Sul. Como resultado deste inventário foi publicado o trabalho "Biodiversidade. Regiões da Lagoa do Casamento e dos Butiazais de Tapes, Planície Costeira do Rio Grande do Sul” (Becker et al. 2007) que abrangeu a comunidade fitoplanctônica, entre elas, as Euglenophyta. Este levantamento revelou alta riqueza de Euglenophyta e carência de estudos mais aprofundados deste grupo de algas na Planície Costeira. Para suprir a lacuna existente foi realizado o estudo taxonômico desta divisão, resultando na publicação dos gêneros: Euglena e Lepocinclis (Alves-da-Silva \& Fortuna, 2006), Phacus (Alves-da-Silva \& Fortuna, 2008) e Strombomonas (Alves-da-Silva \& Kurrle, 2009). Portanto, este é o quinto trabalho da série de estudos da ficoflora de Euglenophyta da Planície Costeira do estado do Rio Grande do Sul.

O país carece de trabalhos abrangendo Euglenophyta incolores, existindo apenas o registro de menos de 30 táxons destas algas em Alves-da-Silva (1998), Bicudo \& Bicudo (1987), Bittencourt-Oliveira (1997), Cecy (1970), Cunha (1913), Menezes (1993), Rosa et al. (1987) e Uherkovich \& Schmidt (1974). Esses flagelados incolores são ainda pouco estudados em nível mundial e nacional, devido a sua raridade, taxonomia problemática e dificuldade na visualização ocasionada pela mobilidade proporcionada por seu(s) flagelo(s) e pela perda do(s) flagelo (s), principalmente em material fixado.

Este estudo tem como objetivo ampliar o conhecimento e distribuição geográfica das algas incolores de Euglenophyta em ambientes aquáticos continentais da Planície Costeira do Rio Grande do Sul e Brasil.

\section{Material e métodos}

O trabalho foi desenvolvido na Planície Costeira do Rio Grande do Sul em margens de 14 ambientes lênticos como banhados, lagoas, açude e canal, em duas áreas localizadas à oeste e leste da Laguna dos Patos: a Lagoa do Casamento e ecossistemas associados $\left(30^{\circ} 03^{\prime}-30^{\circ} 34^{\prime} \mathrm{S}\right.$ e $\left.50^{\circ} 25^{\prime}-50^{\circ} 47^{\prime} \mathrm{W}\right)$ e ecossistemas próximos á região do Butiazal de Tapes $\left(30^{\circ} 23^{\prime}-30^{\circ} 38^{\prime} \mathrm{S}\right.$ e $51^{\circ} 16^{\prime}-51^{\circ} 29^{\prime} \mathrm{W}$ ) ( Fig. 1) .

Nestas duas regiões prevalece um relevo plano e áreas com ocorrência de intensos processos de deposição de sedimentos. São planícies arenosas, com lagoas, banhados e dunas, com predomínio de atividades de agricultura, campos de pastagem e algumas áreas de silvicultura. Apesar das duas áreas serem próximas apresentam diferenças quanto ao relevo, composição de ambientes e uso da terra (Ramos et al. 2007). O clima local é subtropical de acordo com Conti \& Furlan (2003).

\footnotetext{
Seção de Botânica de Criptógamas, Museu de Ciências Naturais da Fundação Zoobotânica do Rio Grande do Sul, Porto Alegre, RS, Brasil

2 Autor para correspondência: alvesdasilva@fzb.rs.gov.br
} 


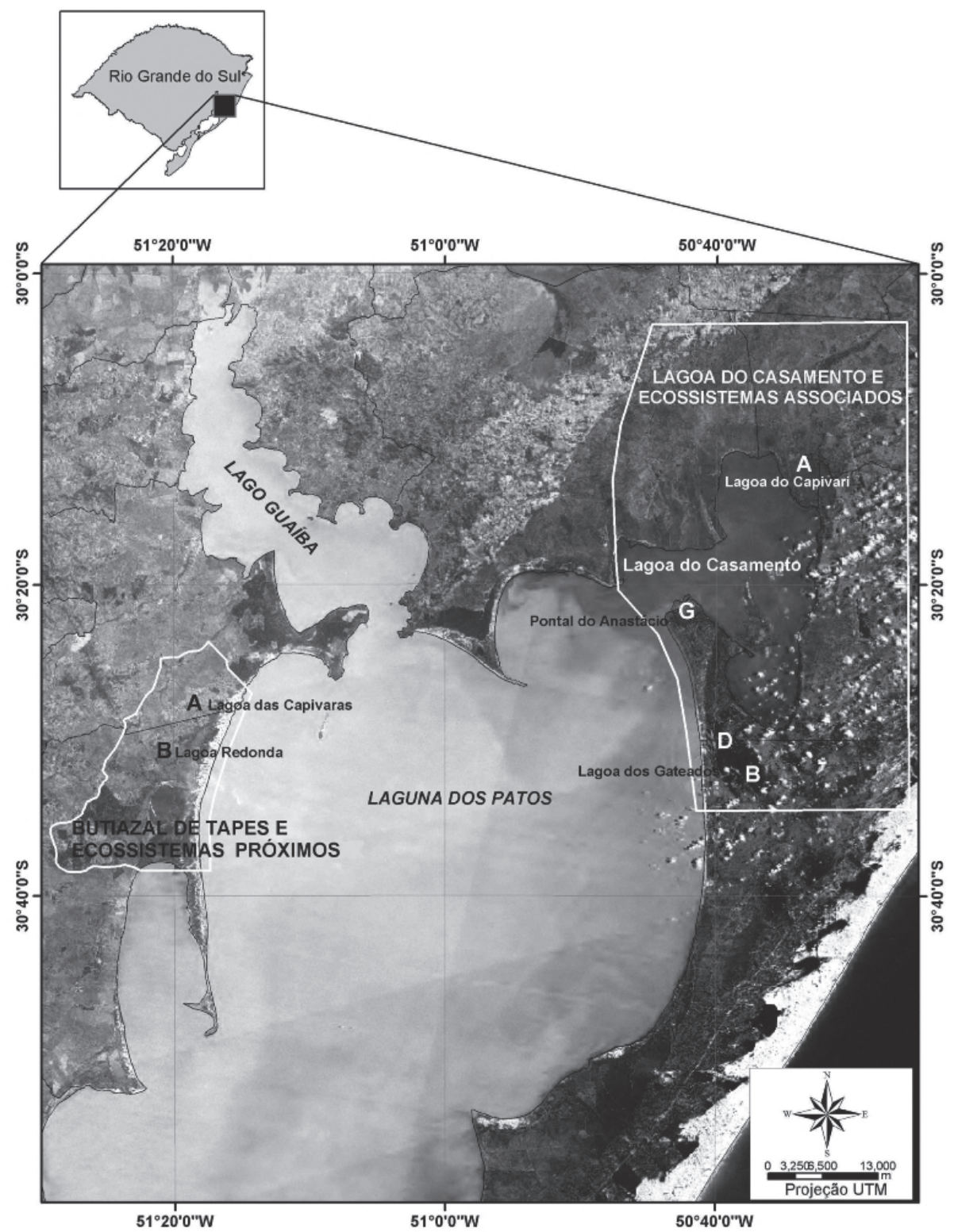

Figura 1. Localização da Lagoa do Casamento e Butiazal de Tapes e seus ecossistemas associados, com indicação das subáreas estudadas em 2003, na Planície Costeira do Rio Grande do Sul, Sul do Brasil.

As amostras foram coletadas através da passagem de rede de plâncton de $25 \mu \mathrm{m}$ de abertura de malha na subsuperfície da água e espremido de macrófitas aquáticas, abrangendo as estações de outono (maio e junho) e primavera (outubro a dezembro) de 2003. As amostras foram conservadas com formaldeído a $3 \%$ e solução de Transeau e encontram-se tombadas no Herbário Prof. Dr.R.H.Schultz (HAS) no Museu de Ciências Naturais da Fundação Zoobotânica do Rio Grande do Sul.

Em campo foram medidas variáveis ambientais como: $\mathrm{pH}$, temperatura, condutividade elétrica e oxigênio dissolvido da água. $\mathrm{O}$ valor do $\mathrm{pH}$ foi determinado por meio de potenciômetro digital Hach, a condutividade elétrica da água através de condutivímetro de campo Hach, a temperatura da água, com termômetro químico e o oxigênio dissolvido através de oxímetro Hach. Em alguns locais o oxigênio não foi medido.

A análise do material vivo e fixado foi realizada entre lâmina e lamínula com auxílio de microscópio óptico e os desenhos realizados com auxílio de câmera-clara acoplada ao microscópio.

São fornecidas informações da variação morfométrica e ilustrações dos táxons, relação entre o comprimento e a largura da célula (Rc/l), comentários e amplitude de algumas variáveis abióticas em que os táxons ocorreram na área de estudo.

Para verificar a similaridade entre as duas áreas estudadas foi aplicado o Índice de Sörensen (IS $=2 \mathrm{c} /(\mathrm{a}+\mathrm{b})$, onde: $\mathrm{a}$ e b são os números de espécies das comunidades a e $b$, respectivamente, e c é o número de espécies comuns às duas comunidades (Brower et al. 1998).

Para identificação dos táxons supra-genéricos foi adotado Leedale (1967). Para a identificação específica e infra-específica foram utilizadas as obras de: Huber-Pestalozzi (1955), Angeler et al. (2002), Skuja (1948), Shi et al. (1999), Bourrelly \& Manguin (1952), Starmach (1983), Popova (1966) e Bourrelly (1970).

\section{Resultados e discussão}

Foram registradas Euglenophyta incolores em somente sete dentre os 14 locais amostrados. A análise de 53 amostras resultou na identificação de nove táxons de Euglenophyta 
incolores distribuídos em quatro ordens: Euglenales, Heteronematales, Rhabdomonadales e Sphenomonadales e em seis gêneros: Cyclidiopsis, Entosiphon, Gyropaigne, Menoidium, Petalomonas e Rhabdmonas, totalizando oito espécies e uma variedade que não a típica.

O gênero melhor representado na Planície Costeira foi Menoidium com o registro de quatro representantes: $M e$ noidium gracile Playf., Menoidium obtusum Pringsheim, M. pellucidum Perty e M. tortuosum (Stokes) Lemm. var. playfairii Bour.

\section{EUGLENALES \\ EUGLENINA}

\section{Cyclidiopsis Korschikow}

Cyclidiopsis acus Korschikow, Travaux de la Biol. Borodinskaja 4: 225, Pl. II, fig. 2, 1917.

Fig. 2-3

Células cilíndricas, 175-202 $\mu \mathrm{m}$ compr., 5-7 $\mu \mathrm{m}$ larg., Rc/1 $=30,5-40$; pólo anterior aquadradado com abertura apical; pólo posterior cônico afilado; paramidos 7-9, bastoniformes; flagelo emergente 1 , ca de $1 / 7 \mathrm{vez}$ o comprimento celular.

Material examinado: BRASIL. Rio Grande do Sul:

Palmares do Sul, 9/V/2003, Werner 104178 (HAS).

Distribuição no Brasil: Primeira citação para o país.

\section{HETERONEMATALES}

HETERONEMATINA

\section{Entosiphon Stein}

Entosiphon polyaulax Skuja, Acta Horti. Bot. Univ. Latv. 7: 42, fig. 29-31. 1932.

Fig. 4-6

Células oblongas a ovadas, 29-36 $\mu \mathrm{m}$ compr., 14-17 $\mu \mathrm{m}$ larg., $\mathrm{Rc} / 1=1,8-2,4$; pólo anterior arredondado levemente acuminado, presença de um sifão; pólo posterior levemente acuminado; película com estrias longitudinais 12; núcleo central a posterior; flagelos 2 , desiguais, o rebocador ca. de $1 / 5 \mathrm{vez}$ do comprimento célula e o outro 1,5 vezes o comprimento celular.

Material examinado: BRASIL. Rio Grande do Sul: Capivari do Sul, 5/V/2003, Werner 104093 (HAS); Palmares do Sul, 8/V/2003, Werner 104136 (HAS).

Distribuição no Brasil: Primeira citação para o país.

\section{RHABDOMONADALES \\ RHABDOMONADINA}

\section{Gyropaigne Skuja}

Gyropaigne kosmos Skuja, Acta Horti Bot. Univ. Latv. 11/12: 113, pl. 7, fig. 16-18. 1939.

Fig. 7-13

Célula ovadas, 32- $43 \mu \mathrm{m}$ compr., 20-28 $\mu \mathrm{m}$ larg., Rc/1 $=1,4-1,6$; pólo anterior arredondado, vista apical estrelada com 8-10 pontas; pólo posterior levemente acuminado; película com estrias levemente dextrógiras; paramidos numerosos, arredondados; núcleo posterior; flagelo ca. de $1 \mathrm{o}$ comprimento celular.

Material examinado: BRASIL. Rio Grande do Sul: Capivari do Sul, 5/V/2003, Werner 104092 (HAS); Capivari do Sul, 5/V/2003, Werner 104093 (HAS); Palmares do Sul, 7/V/2003, Werner 104118 (HAS); Palmares do Sul, 7/V/2003, Werner 104121 (HAS); Palmares do Sul, 29/X/2003, Alves-da-Silva 104362 (HAS); Tapes, 3/ XII/2003, Cardoso 104445 (HAS).

Distribuição no Brasil: Primeira citação para o país.

\section{Menoidium Perty}

Menoidium gracile Playf., Proc. Linn. Soc. N. S. W. 45:137, fig. 4-5. 1921.

Fig. 14

Célula alongada, falciforme, $65-78 \mu \mathrm{m}$ compr., 7-8 $\mu \mathrm{m}$ larg., Rc/1 = 9-10; pólo anterior levemente afilado formando um pequeno rostro, abertura do canal apical; lado ventral côncavo e dorsal convexo; pólo posterior estreito terminado em processo caudal levemente arredondado; película aparentemente lisa; numerosos paramidos bastoniformes; núcleo central, oblongo; flagelo 1 , ca. de $1 / 8$ vez o comprimento celular.

Material examinado: BRASIL. Rio Grande do Sul: Capivari do Sul, 5/V/2003, Werner 104092 (HAS); Capivari do Sul, 5/V/2003, Werner 104093 (HAS); Capivari do Sul, 5/V/2003, Werner 104096 (HAS); Tapes, 3/XII/2003, Cardoso 104445 (HAS).

Distribuição no Brasil: Paraná: Cecy (1990).

Menoidium obtusum Pringsheim, New Phytol. 41: 199, fig. 17, 1942.

Fig. 15-16

Células botuliformes, 41-42 $\mu \mathrm{m}$ compr., $11 \mu \mathrm{m}$ larg., $\mathrm{Rc} / 1=3,7-3,8$; pólo anterior levemente atenuado, rostro muito pequeno, abertura apical; pólo posterior arredondado; paramido 2, de bastoniforme a oblongo; núcleo posterior; flagelo emergente 1 , ca. de $1 / 2$ vez o comprimento celular.

Material examinado: BRASIL. Rio Grande do Sul: Palmares do Sul, 8/V/2003, Werner 104136 (HAS).

Distribuição no Brasil: São Paulo: Pringsheim in HuberPestalozzi (1955).

Menoidium pellucidum Perty, Kenntnis Kleinster Leb. pl. XV, fig. 19, 1852.

Fig. 17

Células falciformes, $48-50 \mu \mathrm{m}$ compr., 6-7 $\mu \mathrm{m}$ larg., Rc/l= 6,8-8; pólo anterior levemente atenuado terminado em rostro; lado ventral levemente côncavo e dorsal levemente convexo; pólo posterior arredondado; paramidos 2; bastoniforme, 12 $\mu \mathrm{m}$ compr.por $5 \mu \mathrm{m}$ larg.; núcleo e flagelo não observados. 


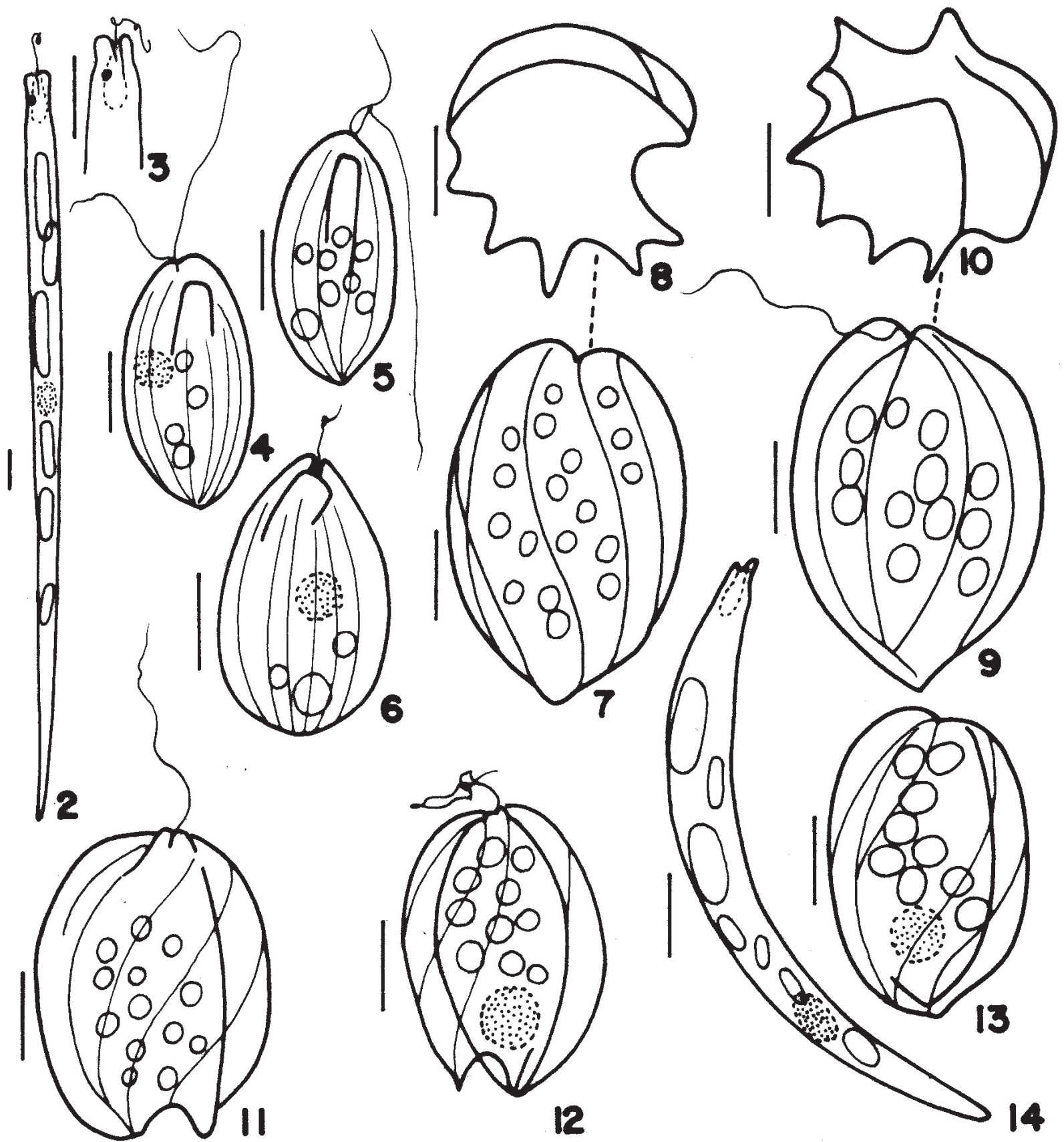

Figura 2-14. 2-3. Cyclidiopsis acus Korschikow, 3- detalhe do pólo anterior. 4-6. Entosiphon polyaulax Skuja. 7-13. Gyropaigne kosmos Skuja, 8 e 10. vista apical. 14. Menoidium gracile Playfair. Escala $=10 \mu \mathrm{m}$.

Material examinado: BRASIL. Rio Grande do Sul: Capivari do Sul, 5/V/2003, Werner 104093 (HAS); Tapes, 3/XII/2003, Cardoso 104445 (HAS).

Distribuição no Brasil: Amazônia: Bittencourt-Oliveira (1997). Rio de Janeiro: Cunha (1913)

Menoidium tortuosum (Stokes) Lemm. var. playfairii Bour.

In. Bourrelly \& Manguin, Alg. Guad. 187, fig. 333. 1952.

Fig. 18-22
Célula falciforme alongada, lado ventral levemente convexo e dorsal levemente concâvo, 23-29 $\mu \mathrm{m}$ compr., 2,5-3,5 $\mu \mathrm{m}$ larg., Rc/1 = 7,4-9,2; pólo anterior levemente atenuado, rostro muito pequeno; pólo posterior atenuado, com processo caudal torcido; paramidos 3-5; bastoniforme a elíptico; núcleo e flagelo não observados.

Material examinado: BRASIL. Rio Grande do Sul: Capivari do Sul, 5/V/2003, Werner 104093 (HAS).

Distribuição no Brasil: Primeira citação para o País. 


\section{SPHENOMONADALES \\ SPHENOMONADINA}

\section{Petalomonas Stein}

Petalomonas platyrhyncha Skuja, Symb. Bot. Uppsal. 9: 217, pl. 26, fig. 1-7. 1946-1948.

Fig. 23-26

Células ovadas, $43-53 \mu \mathrm{m}$ compr., $26-31 \mu \mathrm{m}$ larg., $\mathrm{Rc} / 1=$ 1,6-2,0; pólo anterior acuminado, abertura flagelar subapical, lado dorsal convexo, lado ventral plano-convexo; vista apical com três pontas; pólo posterior termina em processo caudal cônico, 3-7 $\mu \mathrm{m}$ compr.; película com 6 quilhas longitudinais, convergentes nos dois pólos; numerosos paramidos, arredondados; núcleo central a lateral; flagelo não observado.

Material examinado: BRASIL. Rio Grande do Sul: Capivari do Sul, 5/V/2003, Werner 104092 (HAS); Capivari do Sul, 5/V/2003, Werner 104093 (HAS); Capivari do Sul, 5/V/2003, Werner 104096 (HAS); Palmares do Sul, 7/V/2003, Werner 104127 (HAS); Palmares do Sul, 8/V/2003, Werner 104136 (HAS); Palmares do Sul, 9/V/2003, Werner 104174 (HAS); Palmares do Sul, 9/V/2003, Werner 104179 (HAS); Tapes, 3/VI/2003, Torgan 104201 (HAS); Tapes, 4/VI/2003, Torgan \& Werner 104207 (HAS); Tapes, 4/VI/2003, Werner 104213 (HAS); Tapes, 4/VI/2003, Torgan \& Werner 104220 (HAS); Tapes, 4/VI/2003, Torgan \& Werner 104230 (HAS); Palmares do Sul, 29/X/2003, Alves-da-Silva 104362 (HAS); Tapes, 2/XII/2003, Cardoso 104425 (HAS); Tapes, 2/XII/2003, Cardoso 104431 (HAS); Tapes, 3/XII/2003, Alves-da-Silva 104435 (HAS); Tapes, 3/XII/2003, Cardoso 104445 (HAS).

Distribuição no Brasil: Rio Grande do Sul: Alves-daSilva (1998).

\section{Rhabdomonas Fresenius}

Rhabdomonas mirabilis (Playf.) Schroeckh, Lee \& Patterson, Hydrobiologia 493, fig. 9h e 10 a-k. 2003.

Fig. 27-29

Células elípticas a cilíndricas, 23-25 $\mu \mathrm{m}$ compr., 13-15 $\mu \mathrm{m}$ larg., $\mathrm{Rc} / 1=1,5-1,7$; pólos arredondados; abertura do canal apical a subapical; 5-6 estrias espiraladas, proeminentes; vários paramidos, arredondados; núcleo posterior; flagelo 1 .

Material examinado: BRASIL. Rio Grande do Sul: Capivari do Sul, 5/V/2003, Werner 104093 (HAS).

Distribuição no Brasil: Primeira citação para o país.

Esta espécie foi descrita por Playfair (1921) para Austrália como Sphenomonas mirabilis, no entanto, em sua descrição original este autor refere à espécie como possuindo somente um curto flagelo, sem mencionar um segundo flagelo. Schroeckh et al. (2003) com base na presença de somente um flagelo realizaram uma nova combinação transferindo esta espécie para o gênero Rhabdomonas.

Os indivíduos observados apresentaram menores dimensões que as citada por Schroeckh et al., 2003 (30-35 $\mu \mathrm{m}$ compr.) porém a morfologia, as estrias bem marcadas e projetadas dando a impressão do citoplasma retraído na região central da célula conferem com a descrição da espécie.

O estudo permitiu concluir que a conservação das amostras provoca a perda do(s) flagelo(s) o que dificulta a identificação de alguns táxons, por esta razão a identificação das Euglenophyta incolores foi baseada primordialmente em amostras vivas, mas devido ao seu rápido deslocamento foram utilizadas amostras fixadas também, para confirmação das identificações e confecção dos desenhos.

O índice de Sörensen revelou uma similaridade média entre as duas áreas estudadas (0,615, Tab. 1$)$. Apesar da proximidade das duas áreas, a Lagoa do Casamento apresentou maior número de táxons, destacando-se o banhado entre a Lagoa do Capivari e Lagoa do Casamento por ter sido encontrado $89 \%$ dos táxons identificados. Os táxons foram mais freqüentes em ambientes rasos ou que apresentaram macrófitas aquáticas que ao se decomporem contribuíram para o aumento de matéria orgânica e propiciaram a presença destes organismos, resultados que concordam com Wetzel (1993), que cita que as Euglenophyta são mais encontradas em águas pouco profundas e ricas em matéria orgânica.

A estação do ano com maior registro de táxons foi o outono (Tab. 2), possivelmente resultado do revolvimento da coluna da água provocada pela pluviosidade ocorrida durante o período de amostragem e conseqüente deslocamento dos indivíduos do sedimento para superfície. Esses dados corroboram a afirmação de Margalef (1983) que numerosos representantes de euglenofíceas incolores podem viver nas camadas superficiais de sedimentos de lagos.

Petalomonas platyrhyncha foi a espécie com maior distribuição ocorrendo em diferentes ecossistemas como lagoas, banhado e lagoinha entre dunas, enquanto Cyclidiopsis acus e Rhabdomonas mirabilis só ocorreram em um ambiente, respectivamente em canal e banhado. Foram considerados táxons raros por terem sido registrados em uma ou duas amostras: Cyclidiopsis acus, Entosiphon polyaulax, Menoidium obtusum, M. pellucidum, $M$. tortuosum var. playfairii e Rabdomonas mirabilis.

Gyropaigne kosmos, Menoidium gracile, Menoidium pellucidum e Petalomonas platyrhyncha foram espécies encontradas nas duas áreas (Tab. 1).

Apesar de Gyropaigne kosmos ter ocorrido em apenas em dois ambientes foi a espécie que apresentou maior densidade relativa com até oito indivíduos por lâmina.

As Euglenophyta incolores foram encontradas em águas levemente ácidas com $\mathrm{pH}$ variando de 6,1-6,7, e com ampla variação de temperatura $\left(15,7-34,0^{\circ} \mathrm{C}\right)$, condutividade elétrica desde baixa até alta $\left(17,1-362,0 \mathrm{mS} \mathrm{cm}^{-1}\right)$ e oxigênio dissolvido desde muito baixo $\left(<3,0 \mathrm{mg} \mathrm{L}^{-1}\right)$ até alto $(11,0$ mg L ${ }^{-1}$ ) (Tab. 3).

Petalomonas platyrhyncha foi a espécie que tolerou a maior amplitude de variação de $\mathrm{pH}$, condutividade elétrica, oxigênio dissolvido e temperatura da água, ocorrendo em vários ecossistemas aquáticos e apresentando ampla tolerância das condições ambientais analisadas (Tab. 3). Apesar 


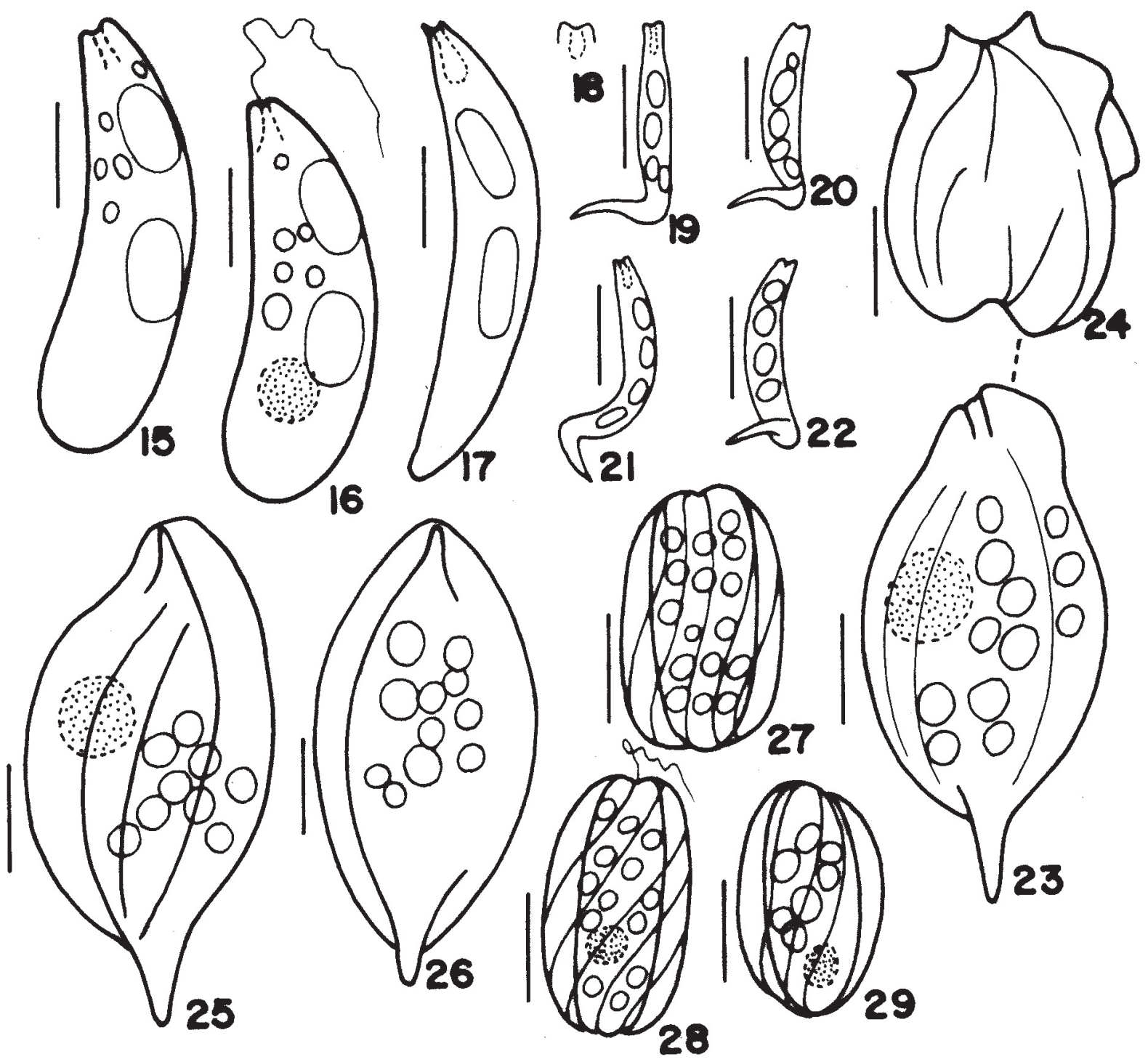

Figura 15-29. 15-16. Menoidium obtusum Pringsheim. 17. Menoidium pellucidum Perty. 18-22. Menoidium tortuosum (Stokes) Lemmermann var. playfairii Bourrelly, 18. detalhe do pólo anterior. 23-26. Petalomonas platyrhyncha Skuja, 24. vista apical. 27-29. Rhabdomonas mirabilis (Playfair) Schroeckh, Lee \& Patterson. Escala $=10 \mu \mathrm{m}$.

Tabela 1. Distribuição dos táxons de Euglenophyta incolores registrados nas duas áreas da Planície Costeira do Rio Grande do Sul, Brasil, no outono e primavera de 2003.

\begin{tabular}{lcc}
\hline Táxons identificados & Lagoa do Casamento & Butiazal de Tapes \\
\hline Cyclidiopsis acus & $\mathrm{X}$ & - \\
Entosiphon polyaulax & $\mathrm{X}$ & - \\
Gyropaigne kosmos & $\mathrm{X}$ & $\mathrm{X}$ \\
Menoidium gracile & $\mathrm{X}$ & $\mathrm{X}$ \\
Menoidium obtusum & $\mathrm{X}$ & - \\
Menoidium pellucidum & $\mathrm{X}$ & $\mathrm{X}$ \\
Menoidium tortuosum var. playfairii & $\mathrm{X}$ & - \\
Petalomonas platyrhyncha & $\mathrm{X}$ & $\mathrm{X}$ \\
Rhabdomonas mirabilis & $\mathrm{X}$ & - \\
Total & 9 & 4 \\
\hline
\end{tabular}


Tabela 2. Distribuição dos táxons de Euglenophyta incolores na Planície Costeira do Rio Grande do Sul, Brasil, em diferentes ambientes das áreas da lagoa do Casamento (LC) e do Butiazal de Tapes (BT), em 2003. Abreviatura: Out/Prim = táxons registrados no outono e primavera.

\begin{tabular}{|c|c|c|c|c|c|c|}
\hline Áreas/ Locais/ Estação do ano/ Táxons identificados & $\begin{array}{l}\text { LC } \\
\text { Lagoa }\end{array}$ & $\begin{array}{c}\text { LC } \\
\text { Banhado }\end{array}$ & $\begin{array}{l}\text { LC } \\
\text { Canal }\end{array}$ & $\begin{array}{c}\text { BT } \\
\text { Lagoinha entre dunas }\end{array}$ & $\begin{array}{l}\text { BT } \\
\text { Açude }\end{array}$ & Estação do ano \\
\hline Cyclidiopsis acus & - & - & $\mathrm{X}$ & - & - & Outono \\
\hline Entosiphon polyaulax & $\mathrm{X}$ & $\mathrm{X}$ & - & - & - & Out/Prim \\
\hline Gyropaigne kosmos & - & $\mathrm{X}$ & - & $\mathrm{X}$ & - & Out/Prim \\
\hline Menoidium gracile & $\mathrm{X}$ & $\mathrm{X}$ & - & $\mathrm{X}$ & - & Out/Prim \\
\hline Menoidium obtusum & $\mathrm{X}$ & - & - & - & - & Outono \\
\hline Menoidium pellucidum & - & $\mathrm{X}$ & - & $\mathrm{X}$ & - & Out/Prim \\
\hline Menoidium tortosus var. playfairii & - & $\mathrm{X}$ & - & - & - & Outono \\
\hline Petalomonas platyrhyncha & $\mathrm{X}$ & $\mathrm{X}$ & - & $\mathrm{X}$ & $\mathrm{X}$ & Out/Prim \\
\hline \multirow[t]{2}{*}{ Rhabdomonas mirabilis } & - & $\mathrm{X}$ & - & - & - & Outono \\
\hline & 4 & 7 & 1 & 4 & 1 & \\
\hline
\end{tabular}

Tabela 3. Amplitude das variáveis ambientais em que cada táxon das Euglenophyta incolores ocorreu na Planície Costeira do Rio Grande do Sul, Brasil, no outono e primavera de 2003. Abreviatura: NM= não medido.

\begin{tabular}{|c|c|c|c|c|}
\hline Táxons/variáveis ambientais & $\begin{array}{l}\text { Temperatura } \\
\text { da água }\left({ }^{\circ} \mathrm{C}\right)\end{array}$ & $\mathrm{pH}$ & $\begin{array}{l}\text { Condutividade } \\
\left(\mu \mathrm{S} \mathrm{cm}^{-1}\right)\end{array}$ & $\begin{array}{c}\text { Oxigênio dissolvido } \\
\left(\mathrm{mg} \mathrm{L}^{-1}\right)\end{array}$ \\
\hline Cyclidiopsis acus & 24,9 & 6,1 & 17,1 & NM \\
\hline Entosiphon polyaulax & $16-24,4$ & $6,2-6,6$ & $110,0-362,0$ & $6,2-6,8$ \\
\hline Gyropaigne kosmos & $18-27,1$ & $6,4-6,6$ & $110,0-136,0$ & $<3,0-6,8$ \\
\hline Menoidium gracile & 24,4 & 6,6 & 110,0 & 6,8 \\
\hline Menoidium obtusum & 16,0 & 6,2 & 362,0 & 11,0 \\
\hline Menoidium pellucidum & 24,4 & 6,6 & 110,0 & 6,8 \\
\hline Menoidium tortuosum var. playfairii & 24,4 & 6,6 & 110,0 & 6,8 \\
\hline Petalomonas platyrhyncha & $15,7-34,0$ & $6,1-6,7$ & $17,1-362,0$ & $<3,0-9,5$ \\
\hline Rhabdomonas mirabilis & 24,4 & 6,6 & 110,0 & 6,8 \\
\hline
\end{tabular}

de sua distribuição nas duas áreas, sempre foi registrada em baixa densidade relativa com a presença de um a dois indivíduos por lâmina.

Apesar da baixa riqueza, o registro destas algas se reveste de grande importância, visto que, quanto à distribuição, todos os nove táxons de Euglenophyta incolores são novas citações para a Planície Costeira do Estado e cinco são primeiros registros para o Brasil: Cyclidiopsis acus, Entosiphon polyaulax, Gyropaigne kosmos, Menoidium tortuosum var. playfairii e Rhabdomonas mirabilis e ainda, Menoidium gracile, Menoidium obtusum e Menoidium pellucidum são novas citações para o Rio Grande do Sul. O estudo também amplia o conhecimento da distribuição das Euglenophyta incolores para a região subtropical do país.

A Lagoa do Casamento e seus ecossistemas associados estão localizados próximos da bacia Hidrográfica do Lago Guaíba que abrange o maior contingente populacional e concentração de atividade econômicas do Estado. A contribuição antrópica nesta área e a conectividade das lagoas pode ser a provável explicação para maior riqueza de Euglenophyta incolores nestes ecossistemas do que nos verificados na região do Butiazal de Tapes que apresentam ambientes mais protegidos e alguns temporários.

\section{Agradecimentos}

Ao Conselho Nacional de Desenvolvimento Científico e Tecnológico (CNPq) pela concessão de Bolsa de Iniciação Científica (PIBIC) à segunda autora (Proc. $n^{\circ}$ 100246/2007-9). Aos colegas da Seção de Botânica de Criptógamas do Museu de Ciências Naturais da Fundação Zoobotânica do Rio Grande do Sul, pela execução e auxílio nas coletas, à Rejane Rosa, pela cobertura à nanquim dos desenhos e a Ricardo Aranha do Laboratório de Geoprocessamento, pela elaboração do mapa.

\section{Referências bibliográficas}

Alves-da-Silva, S.M. 1998. Levantamento Taxonômico e Variação Temporal das Euglenophyceae de um Reservatório Raso no Município de Triunfo, Estado do Rio Grande do Sul. Tese de Doutorado. Universidade Estadual Paulista, Rio Claro, São Paulo.

Alves-da-Silva, S.M. \& Fortuna, J.R. 2006. Euglenophyceae de ambientes lênticos na planície costeira do Rio Grande do Sul, sul do Brasil: gêneros Euglena Ehr. e Lepocinclis Perty. Acta Botanica Brasilica 20(2): 411-422.

Alves-da-Silva, S.M. \& Fortuna, J.R. 2008. O gênero Phacus (Euglenophyceae) em sistemas lênticos da Planície Costeira do Rio Grande do Sul, sul do Brasil. Acta Botanica Brasilica 22(3): 684-700. 
Alves-da-Silva, S.M. \& Kurrle, I.L.P. 2009. O gênero Strombomonas (Euglenophyceae pigmentadas) em ambientes lênticos na Planície Costeira do Rio Grande do Sul, Brasil. Acta botanica brasilica 23(4): 944-955.

Angeler, D.G.; Schagerl, M. \& Muller, A.N. 2002. Taxonomic comments on the genus Menoidium (Euglenozoa), with a description of Menoidium intermedium sp. nov. European Journal of Protistology 38: 393-404.

Becker, F.G.; Ramos, R.A. \& Moura, L. de A. (orgs.). 2007. Biodiversidade. Regiões da Lagoa do Casamento e dos Butiazais de Tapes, Planície Costeira do Rio Grande do Sul. Brasília, Ministério do Meio Ambiente.

Bicudo, C. E. \& Bicudo, D. C. 1987. Some new and rare Euglenophyce from the state of São Paulo, southern Brazil. Acta Botanica Brasilica 1(1): 43-48.

Bittencourt-Oliveira, M. 1997. Euglenophyceae do Reservatório de Balbina, Estado do Amazonas, Brasil. Hoehnea 24(1): 21-35

Bourrelly, P. 1970. Les algues d'eau douce: initation à la systematique, III. les algues blues et rouges, les Eugléniens, Peridiniens et Cryptomonadines. Paris, N. Boubée.

Bourrelly, P. \& Manguin, E. 1952. Algues d'aeu douce de la Guadaloupe et dépendances. Centre National de la Recherche Scientifique. p. 1-281.

Brower, J.E.; Zar, J.H. \& Van Ende, C.N. 1998. Field and Laboratory Methods for General Ecology, WCB/McGraw.

Cecy, I. I. T. 1990. A restinga do Pontal do Sul, município de Paranaguá, Pr. - I. Levantamento ficológico (Euglenophyta) e físico-químico. Arquivos de biologia e tecnologia 33 (1): 1-79.

Conti, J.B. \& Furlan, S.A. 2003. Geoecologia: o clima, os solos e a biota. Pp. 67-207. In: J.L. Ross (org.). Geografia do Brasil. São Paulo, Ed. da Universidade de São Paulo.

Cunha, A. M. 1913. Contribuição para o conhecimento da fauna de protozoários do Brasil. Memórias do Instituto Osvaldo Cruz 5(2): 101-122.
Huber-Pestalozzi, G. 1955. Euglenophyceen. Pp. 1-605. In: G. HuberPestalozzi (ed.). Das phytoplankton des Susswässers: Systematik und Biologie. Teil 4, E. Schweizerbart'sche Verlangsbuchhandlug, Stuttgart. Leedale, G.F. 1967. Euglenoid Flagellates. New Jersey. Ed. Prentice-Hall. Margalef, R. 1983. Limnologia. Barcelona. Ed. Omega.

Menezes, M. 1993. New records of heterotrofic flagellates (Euglenophyta) from Brasil. Nova Hedwigia 56 (1-2): 131-137.

Popova, T.G. 1966. Euglenophyta. Trachelomonas, Strombomonas, Eutreptia, Euglena. Flora Plantarum Cryptogamarum URSS 3(1): $1-410$

Ramos, R.; Pasqualetto, A.I.; Balbueno, R.A. \& Pinheiro, E. da S. 2007. 5. Paisagem, uso e cobertura da terra. Pp. 61-83. In: F.G. Becker; R.A. Ramos \& L.de A. Moura (orgs.). Biodiversidade. Regiões da Lagoa do Casamento e dos Butiazais de Tapes, Planície Costeira do Rio Grande do Sul. Brasília, Ministério do Meio Ambiente.

Rosa, Z.M., Ungareti, I., Kremer, L.M., Alves-da-Silva, S.M.; Callegaro, V.L. M. \& Werner, V.R. 1987. Ficoflora de ambientes lênticos - estudo preliminar da região de Charqueadas, Rio Grande do Sul, Brasil, com vistas à avaliação ambiental. Acta Botanica Brasilica 1(2): 165-188.

Schroeckh, S.; Lee W. J. \& Patterson, D.J. 2003. Free-living heterotrophic euglenids from freshwater sites in mainland Australia. Hydrobiologia 493: $131-166$

Shi, Z.; Wang, Q.; Xie, S.; Dai, J. \& Chen, L. 1999. Euglenophyta. Flora Algarum Sinicarum Aquae Dulcis, t. 6.

Skuja, H. 1946-1948. Taxonomie dês Phytoplanktons einiger seen in Uppland, Schweden. Uppsala. (Symbolae botanicae upsaliensis, 9)

Starmach, K. 1983. Euglenophyta. In: K. Starmach. Flora Slodkowodna Polski. 3. Warszawa, Polska Academia Nauk.

Uherkovich, G. \& Schmidt, G.W. 1974. Phytoplankton taxa in dem zentralamazonischen Schwemmlandsee. Amazoniana 2: 243-283.

Wetzel, R.G. 1993. Limnologia. 2 ed., Lisboa. Fundação Carlouse Gulbenkian. 